\title{
Responsiveness of Hepatic and Cerebral Cytochrome P450 in Rat Offspring Prenatally and Lactationally Exposed to a Reconstituted PCB Mixture
}

\author{
Patrizia Bonfanti, ${ }^{1}$ Francesca Comelli, ${ }^{2}$ Laura Assi, ${ }^{1}$ Lavinia Casati, ${ }^{3}$ Alessandra Colciago, ${ }^{3}$ \\ Sara Villa, ${ }^{1}$ Angela Santagostino, ${ }^{1}$ Barbara Costa, ${ }^{2}$ Anita Colombo ${ }^{1}$ \\ ${ }^{1}$ Dipartimento di Scienze dell'Ambiente e del Territorio, Università di Milano Bicocca, \\ P.zza della Scienza 1, 20126 Milano, Italy \\ ${ }^{2}$ Dipartimento di Bioscienze, Università di Milano Bicocca, P.zza della Scienza 3, \\ 20126 Milano, Italy \\ ${ }^{3}$ Dipartimento di Endocrinologia, Università di Milano, Milano, Italy
}

Received 12 March 2012; revised 27 July 2012; accepted 4 August 2012

\begin{abstract}
Perinatal polychlorinated biphenyl (PCB) exposures still remain a serious health concern because offspring receive PCB burden from mother during vulnerable processes of development. Since cytochrome P450 (CYP) represents a toxicological endpoint, in the present study, representing an extended investigation of a previous multitasked one, we explored the long-term responsiveness of CYP1A and CYP2B isoforms by Western blot analysis in liver and whole brain of lactating (PN12), weaning (PN21), and adult offspring (PN60) rats prenatally and lactationally exposed to a reconstituted PCB mixture (RM) of noncoplanar PCB138, 153, 180, and coplanar PCB126 congeners. We chose highly chlorinated PCBs instead of lower chlorinated one, because their recalcitrance to biotransformation makes easy their accumulation/persistence in tissues and breast milk. Dioxin-like congener PCB126 binding aryl hydrocarbon receptor (AHR) is responsible of many toxic effects. Pregnant Sprague-Dawley dams with high affinity AHR received subcutaneous injection of RM (10 mg/kg body weight) daily during gestation (days 15-19) and twice a week during breast-feeding. The results evidenced a transfer of PCBs to neonates through milk and a significant responsiveness of hepatic CYP in both mothers and offspring. In liver of exposed progeny, CYP isoforms exhibited a significant increment at PN12 (70\% over control) and at PN21 (270\% over control). Contrary to dams, in adult PCB offspring CYP levels showed a decline up to values similar to those of control. This transient developmental responsiveness of CYP isoforms in offspring liver reflects roughly the time course of hepatic PCB levels previously reported. Even if congeners were detected in brain, we failed in evidencing a responsiveness of CYP isoforms probably because of region-specific CYP expression in this organ. In conclusion, induction of offspring hepatic CYP is index of liver PCB burden, and despite the insensitivity of whole brain CYP we cannot exclude brain vulnerability toward PCB. (c) 2012 Wiley Periodicals, Inc. Environ Toxicol 29: 856-866, 2014.
\end{abstract}

Keywords: PCB153; PCB138; PCB180; PCB126; lactation; rat offspring; cytochrome P450

Correspondence to: P. Bonfanti; e-mail: patrizia.bonfanti@unimib.it

Contract grant sponsor: Italian MIUR.

Contract grant number: PRIN 2004.

Published online 15 September 2012 in Wiley Online Library (wileyonlinelibrary.com). DOI 10.1002/tox.21812

\section{INTRODUCTION}

Polychlorinated biphenyls (PCBs) are an important group of environmental pollutants that had been extensively used in numerous industrial products until the 1970s, and 
subsequently banned worldwide because of their toxicity. Despite this measure, these contaminants still persist ubiquitously in the environment in consequence of their chemical stability. Moreover, the lipophilic nature and resistance of PCBs toward biotransformation give rise to their accumulation and biomagnification in the food chain and to their presence in all the biological matrices including human adipose tissue, blood, and milk (Gallenberg and Vodicnik, 1989).

One of the most serious health concerns from PCB environmental contamination is the exposure during the critical period of development, when the processes of differentiation and maturation of immune, neurological, and physiological systems make fetus and neonate particularly vulnerable (Eriksson et al., 2006; Cocchi et al., 2009; Colciago et al., 2009).

Although PCBs are transferred through placenta to the fetus already during gestation (Mitsuro et al., 2005; Bonfanti et al., 2009), milk, with its high lipid content, can represent a concentrated delivery mechanism of PCBs to neonates (Dekoning and Karmaus, 2000; Filho et al., 2009). A number of human epidemiologic and animal experimental studies have established an association between PCB exposure and neurodevelopmental and neurobehavioral deficits (Eriksson et al., 2006; Roegge and Schantz, 2006; Piedrafita et al., 2008; Colciago et al., 2009).

It is still debated if PCBs exert their toxicity by producing hormonal perturbations or by binding cytosolic receptors that act as transcription factors responsible to mediate several of the effects induced by these compounds. The binding to cytosolic receptors produces a well-known biochemical effect that is the induction of cytochrome P450 (CYP), an enzyme superfamily that catalyzes the detoxification or bioactivation of xenobiotics. The pattern of chlorine substitutions in the two phenyl rings gives each $\mathrm{PCB}$ congener the ability to induce CYP-specific isoforms: coplanar or dioxin-like PCBs enhance CYP1 family expression (i.e., CYP1A) through the binding to the aryl hydrocarbon receptor (AHR), while noncoplanar PCBs have been shown to increase expression of CYP2 (i.e., CYP2B) and/or CYP3 (i.e., CYP3A) families particularly in mammals (Safe, 1994) through the constitutive androstane receptor and pregnane-X-receptor, respectively. In particular, PCBs with two ortho chlorine substitutions (i.e., PCB153 and 180) have been reported to induce preferentially $\mathrm{CYP} 2 \mathrm{~B}$ isoforms, whereas congeners with more than two ortho chlorines (i.e., PCB155) are efficacious inducers of CYP3A in hepatocyte cultures and in rats (Schuetz et al., 1986).

Although a direct role of these CYP isoforms in PCB toxicity has yet to be firmly established (Rifkind, 2006), it is unquestionable whether the CYP-increased expression highlights the biological activity of these pollutants. Alterations in constitutive levels of CYP isoform expression assume a toxicological significance even more during prenatal and postnatal development, when CYP isoform expression undergoes significant physiological changes (Jorhi et al., 2008). To the best of our knowledge, limited studies are presently available on long-term effect of perinatal PCB exposure on the expression of CYP isoforms, in particular in relation to their toxicokinetic behavior (Curran et al., 2011).

Previously, we have carried out a multitasked study in which we have evaluated PCB accumulation in liver and brain along with the measurements of many morpho-functional parameters from birth to adulthood in the same groups of rats exposed in utero and via breast milk to a reconstituted mixture (RM) of four "indicator congeners", selected among the most abundant in biological matrices, of which three noncoplanar congeners (PCB138, 153, and 180), and a coplanar congener (PCB126; Bonfanti et al., 2009; Cocchi et al., 2009; Colciago et al., 2009). We chose a mixture of highly chlorinated PCBs instead of lower molecular weight one, because their recalcitrance to biotransformation makes easy their accumulation and persistence in tissues and biological matrices such as breast milk (Ramos et al., 1997; Bachour et al., 1998; Lanting et al., 1998). PCB126, even if detected in trace in biological matrices, was selected because as dioxin-like congener is able to bind AHR, whose activation mediates many toxic effects.

To highlight the AHR-mediated effects in our studies, we chose a Sprague-Dawley rat strain (CD SPF/VAF) with a high affinity AHR since a study by Curran et al. (2011) demonstrates the importance of receptor binding affinity in regulating the pharmacokinetics of coplanar PCBs.

The exposure to the RM covered the last period of gestation and all lactation interval (from gestation day 15 until postnatal day 21), which in many mammal species coincide with rapid growth, development, and sex differentiation of the brain (Eriksson et al., 2006; Colciago et al., 2009). Last of all, we chose a dose sufficiently low not to cause embryotoxicity but high enough to be effective in inducing longlasting toxic effects detectable also in adult offspring.

All the results from our multitasked study makes it clear that developmental exposure to the four selected PCB compounds produced important long-lasting changes in offspring such as alteration of dimorphic hypothalamic expression of the main enzymes involved in sex steroid metabolite formation, disregulation of hypothalamic-pituitary growth hormone $(\mathrm{GH})$ axis, which controls $\mathrm{GH}$ secretion, in terms of increased somatostatin expression in hypothalamic periventricular nucleus (both males and females) and lateral arcuate nucleus (males only) and decreased GH mRNA levels in the pituitary of male rats, a significant reduction of bone mineral content and cortical bone thickness of tibiae in male rats joint to increased width of the epiphyseal cartilage disk (Cocchi et al., 2009), as well as impairment of sexual behavior and spatial learning (tested with Morris water maze method) and memory retention (passive avoidance test) specifically in males (Colciago et al., 2009).

Moreover, another study from our laboratory has demonstrated that no induction of CYP1A1/2 (induced by the 
coplanar PCB126) and CYP2B1/2 (induced by the phenobarbital type inducers PCB153 and 180), as evaluated by Western blotting, appears in fetal liver and brain at gestational day (GD) 20 even if a transplacental transfer of the selected congeners occurred as demonstrated by bioaccumulation data (Bonfanti et al., 2009).

In the current study, we intended to investigate the responsiveness and the temporal variations in expression profile of CYP1A and CYP2B protein isoforms, in the two target tissues liver and brain of pups at postnatal day (PN) 12, weaning (PN21) and young adult males and females (PN60) of Sprague-Dawley rat (CD SPF/VAF) following prenatal and lactational exposure to the RM above reported. Moreover, since milk is the way of contamination for offspring, the transfer ratio for each analyte from adipose tissue of dam to milk and from milk to the tissues of pups at PN12 were evaluated. These data will be useful to determine in offspring the transfer rate of these contaminants via suckling and to highlight the sensitivity of CYP induction as a predictive method to evaluate the toxicity of the RM congeners during the susceptible postnatal period.

\section{MATERIALS AND METHODS}

\section{Chemicals}

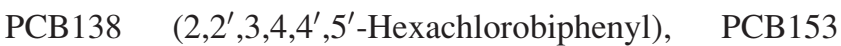

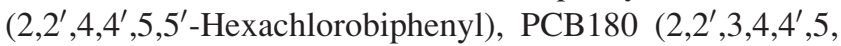
$5^{\prime}$-Heptachlorobiphenyl), PCB126 (3,3',4,4',5-Pentachlorobiphenyl) [purity $100 \%$ ], present in the mixture, were supplied by Chemical Research (Rome, Italy), PCB30, 40, 128 , and 141 , used as internal or recovery standards, were supplied by Dr. Esternstorfer (Augsburg, Germany). All solvents (residue analysis grade, Merck, Settimo Milanese, Italy) were checked by gas chromatography (GC) before use. Sulfuric acid superpure for trace analysis was purchased from Carlo Erba Reagents (Arese, Italy). Prepacked florisil columns (Ultraclean SPE Florisil $500 \mathrm{mg} / 4 \mathrm{~mL}$ ) were purchased by Alltech (Sedriano, Italy). Cellulose extraction thimbles for Soxhlet were purchased by Albet (Barcelona, Spain). Protease inhibitor cocktail and the enhanced chemiluminescence detection kit were purchased from Roche (Monza, Italy). The polyclonal antibodies against CYP1A1/2 and CYP2B1/2 were purchased from Chemicon (DBA, Segrate, Italy). All other chemicals were obtained from Sigma (Milan, Italy).

\section{PCB Mixture}

The reconstituted PCB mixture (RM) was composed by PCB138, 153 (both hexachlorobiphenyls), and 180 (heptachlorobiphenyl) at the same concentration (each representing one-third of the total), while PCB126 (coplanar pentachlorobiphenyl), normally present in trace in biologi- cal extracts, was added to the total mixture in a $1 / 10000$ ratio. These congeners were dissolved in peanut oil $(20 \mathrm{mg}$ mixture $/ \mathrm{mL}$ oil) in order to prepare a solution suitable for subcutaneous injection. The subcutaneous route was used to ensure complete absorption and a systemic distribution of RM in maternal tissues.

\section{Animals and Treatment}

The work presented in this article represents the analysis of biological samples collected during the course of a study aiming to evaluate the alterations of neuroendrocrine, metabolic, and behavioral functions in offspring exposed to PCB RM during gestation and lactation. The details of treatment, performed in the Department of Endocrinology Animal Facility, University of Milan, as well as results of birth outcomes, litter parameters, and other investigations have been reported in previously published articles (Bonfanti et al., 2009; Cocchi et al., 2009; Colciago et al., 2009).

Briefly, nulliparous female (3-5 months) of SpragueDawley rats (CD SPF/VAF, Charles Rivers, Calco, Italy) were housed individually in clean polypropylene cages and maintained in a controlled conditions (environmental temperature, $21-23^{\circ} \mathrm{C}$; light schedule: $14 \mathrm{~h}$ light- $10 \mathrm{~h}$ dark). All animals were fed with a standard pellet diet and tap water ad libitum.

Sperm-positive vaginal smears were considered as day 0 of gestation (GD0). Pregnant rats $(n=30)$ were allocated randomly into two groups. Dams in group 1 received subcutaneous injection of RM $10 \mathrm{mg} / \mathrm{kg}$ body weight/die $(0.5$ $\mathrm{mL} / \mathrm{kg}$; corresponding to $3.333 \mathrm{mg} / \mathrm{kg}$ body weight $/$ die for each noncoplanar congener and $1 \mu \mathrm{g} / \mathrm{kg}$ body weight/die for PCB126) from GD15 to GD20; dams in group 2 served as control and received peanut oil in an identical manner. All the dams deliver spontaneously and during breast-feeding until PN 21 were treated with the same dose, twice a week (in particular at PN3, PN6, PN10, PN13, PN17). From each group, five dams and their litters were sacrificed at PN12 and PN21, respectively. After weaning the remaining litters were reared for sex, housed separately (four rats for each litter per cage), and young adults (PN60) with their mothers were sacrificed.

All analysis were performed on all dams of each experimental time point and on single offspring female $(n=3)$ and male $(n=3)$ randomly selected from each litter.

\section{PCB Quantification}

Samples of breast milk (PN12) obtained from pup gastric contents and dam mesenteric adipose tissue were collected from different individuals, snap-frozen in liquid nitrogen and stored at $-80^{\circ} \mathrm{C}$ until determination of PCB content by gas chromatography/mass spectrometry as previously described in Cocchi et al. (2009). Briefly, all samples were 
homogenized with sodium sulfate anhydrous, spiked with 25 ng of PCB40 and 128 to check recovery efficiencies of methodology and Soxhlet extracted for $24 \mathrm{~h}$ in $n$-hexane. The extracts were reduced in a rotary evaporator under vacuum to $5 \mathrm{~mL}$, concentrated in a vial under a gentle nitrogen flow to $2 \mathrm{~mL}$, then an equal volume of sulfuric acid was added, vortexed for $5 \mathrm{~min}$ and let it stand for $24 \mathrm{~h}$. Hexane layers were pipetted off and charged on Ultraclean SPE Florisil, which was eluted with $8 \mathrm{~mL}$ of ethylacetate: hexane (1:9) and $1 \mathrm{~mL}$ of hexane. The sample, reduced under gentle stream of nitrogen to $0.5 \mathrm{~mL}$, was transferred to $2 \mathrm{~mL} \mathrm{GC}$ vials. All extracts were further concentrated to facilitate solvent exchange to $25 \mu \mathrm{L}$ of dodecane containing two internal standards (PCB30 and 141) for subsequent PCB quantification analysis. Each extract were analyzed in triplicate by GC (gas chromatograph Agilent 6890N equipped with a 50 mCPSil8 column $0.25 \mathrm{~mm}$ i.d. and $0.25-\mu \mathrm{m}$ film thickness), coupled with Mass Selective Detector (Agilent MSD $5973 \mathrm{~N})$, positive electron impact $(\mathrm{EI}+)$ source in selected ion monitoring (SIM) mode. Samples $(2 \mu \mathrm{L})$ were injected by an Agilent 7683 auto injector, with the injection port at $250^{\circ} \mathrm{C}$ in splitless mode. The carrier gas was helium, and the flow rate $1 \mathrm{~mL} / \mathrm{min}$. The oven program was: $100^{\circ} \mathrm{C}$ for 2 $\min , 20^{\circ} \mathrm{C} / \mathrm{min}-140^{\circ} \mathrm{C}, 4^{\circ} \mathrm{C} / \mathrm{min}-200^{\circ} \mathrm{C}, 200^{\circ} \mathrm{C}$ for 13 $\min , 4^{\circ} \mathrm{C} / \mathrm{min}-300^{\circ} \mathrm{C}, 300^{\circ} \mathrm{C}$ for $10 \mathrm{~min}$. The quadrupole MS was set in SIM mode, with an EI+ source, a source temperature of $250^{\circ} \mathrm{C}$, interface temperature of $280^{\circ} \mathrm{C}$. The mean recovery of each analyte ranged between 70 and $110 \%$, and reproducibility was calculated by means of a triplicate analysis giving an overall $10 \%$ error. A series of procedural blanks were analyzed periodically. Reported values were averaged for the measure of analytical variance and blank-corrected. Method detection limit was determined as the instrument detection limit of the lowest concentration standard of each analyte, which was $0.5 \mathrm{pg} / \mu \mathrm{L}$ for each congener. Analytical (instrument) variation is typically $<10 \%$ as measured by repeated injections of samples.

\section{Microsome Preparation for Cytochrome P450 Analysis}

As previously described in Bonfanti et al. (2009), fragments of about $0.5 \mathrm{~g}$ from livers and brains of different individuals both for dams and offspring were homogenized in ice-cold $0.15 \mathrm{M} \mathrm{KCl}(\mathrm{v}: \mathrm{w})$, centrifuged at $9000 \times \mathrm{g}$, at $4^{\circ} \mathrm{C}$ for 10 min and the collected supernatants were then ultracentrifuged at $100000 \times g$, at $4^{\circ} \mathrm{C}$ for $1 \mathrm{~h}$ in order to obtain microsomal pellets that were stored at $-80^{\circ} \mathrm{C}$ until further biochemical analysis.

The brain samples were collected as follows: the hypothalamic areas from each dam and pup were separated from the entire brain to assess specific parameters (see Cocchi et al., 2009; Colciago et al., 2009) and processed separately. The remaining tissue (cerebellum included) was used for the purpose of this work.

\section{Spectrophotometric Cytochrome P450 Assay}

The hepatic total content of CYP was determined spectrophotometrically in livers and brains according to Omura and Sato (1964). Briefly, microsomal pellets were dissolved in Tris- $\mathrm{HCl}$ buffer $0.1 \mathrm{M} \mathrm{pH} 7.4(1: 1 \mathrm{w}: \mathrm{v})$ and then diluted $1: 10$ in the same buffer. Few crystals of solid sodium dithionite (Sigma Aldrich, Milano, Italy) were added to 2 $\mathrm{mL}$ of microsomal suspension and the sample was gently saturated with bubbles of carbon monoxide for $\sim 60 \mathrm{~s}$. The reduced-CO spectrum was recorded from 400 to $500 \mathrm{~nm}$ using a Jasco V-530 spectrophotometer. The specific content of CYP (nmol/mg protein) was calculated according to Lambert-Beer's Law using an extinction coefficient value for CYP of $0.091 \mathrm{nM} / \mathrm{cm}$.

\section{SDS-PAGE and Immunoblotting}

Aliquots of each microsomal sample were used for protein determination according to Lowry et al. (1951). Microsomal proteins for CYP1A1/2 and CYP2B1/2 isoforms (40 $\mu \mathrm{g} /$ well), obtained from individual dams and offspring were mixed with sample buffer, loaded onto $10 \%$ SDSpolyacrylamide gel, separated electrophoretically at a constant voltage $(120 \mathrm{~V}, 0.007 \mathrm{~A})$ and then transferred onto a nitrocellulose membrane (Schleicher \& Schuell, BAS 85, Dassel, Germany) with the semidry method for $90 \mathrm{~min}$ at room temperature. The membrane was incubated with 5\% nonfat dry milk in PBST (16 mM Na $2 \mathrm{HPO}_{4}, 1.9 \mathrm{mM}$ $\mathrm{NaH}_{2} \mathrm{PO}_{4}, 6.7 \mathrm{M} \mathrm{NaCl}, \mathrm{pH} 7.5,0.1 \%$ Tween 20; blocking solution) overnight at $4{ }^{\circ} \mathrm{C}$ and then incubated with primary polyclonal antibody directed against rat CYP1A1/2 or CYP2B1/ 2 cytochrome P450 (Chemicon International, Temecula, CA) diluted 1:1000 in blocking solution, at $37^{\circ} \mathrm{C}$ for $2 \mathrm{~h}$. After washing in PBST buffer, the blot was incubated with secondary antibody (anti-rabbit IgG, peroxidase linked $\mathrm{F}\left(\mathrm{ab}^{\prime}\right) 2$ fragment, 1:1500 in 3\% blocking solution) for $1 \mathrm{~h}$ at room temperature. After washing in PBST buffer, the blot was detected with an enhanced chemiluminescence detection kit. At least two independent blots for each liver and brain sample (dams $n=5$ for each group and offspring $n=6$ for each dam) were performed and the quantification of the signal was analyzed by the image analysis software ImageJ (Scion Corporation, Frederick, MD). Any change in protein levels was expressed as percentage over control.

\section{Statistical Analysis}

All values are represented as mean \pm SEM. Data were statistically analyzed by the Statgraphics plus program for Windows (version 5.1, MD) or by GraphPad Prism 4. ANOVA and the Duncan Multiple Range test were applied when normality and homogeneity of variance in several distributions of investigated parameters were satisfied. Data, which did not conform to the assumptions, were analyzed 

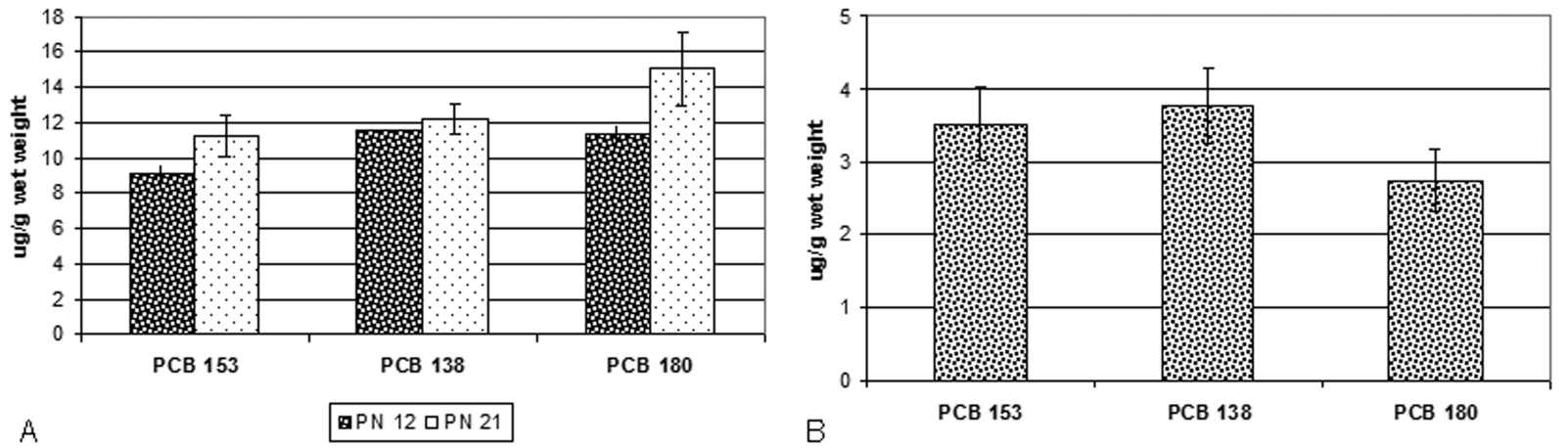

Fig. 1. Concentrations of each PCB congener in mesenteric adipose tissue of dams PN12 and PN21 (A) and in milk obtained by offspring stomach at PN12 (B) measured by gas chromatography/mass spectrometry. Statistical differences were evaluated with Duncan's multiple comparison after ANOVA. Results are reported as mean \pm SEM $(n=5$ dams, $n=3$ offspring female, and $n=3$ offspring male at considered experimental point).

by the Kruskal-Wallis test (a test for differences of location in ranked data grouped by single classification) and the Dunns test for comparing pair of groups as post-test.

\section{RESULTS}

\section{Levels of PCB Congeners in Maternal and Offspring Samples}

PCB levels were analyzed in adipose tissue of dams at PN12 and PN21 and in milk at PN12. All other PCB level data in maternal and offspring liver and brain, used to calculate the transfer rates from mothers to milk and from milk to pup, were reported in our previous articles (Cocchi et al., 2009).

None of the dosed congeners were found in any batches of sample blanks, representing the level of reagent and laboratory contamination, as well as in all control samples. Detectable amounts of PCB 153, 138, and 180 were identified in all examined maternal and offspring tissues, while PCB 126 concentration was less than the detection limit in all samples.

The quantification of PCBs in dam mesenteric adipose tissue has evidenced that congener levels differed slightly over the two time intervals considered. In particular, PCB180 concentration measured at the middle of lactation period (PN12) increased at weaning (PN21), even if this variation was not statistically significant [Fig. 1(A)].

Since milk represents a PCB elimination route for dams and also gives a good estimate of exposure of breast-feeding infants, we wished to investigate the contamination of milk sampled at PN12. In this matrix PCB 153, 138, and 180 levels resulted to be considerable being only 2.5 - to 4-fold lower than those in dam mesenteric adipose tissue [Fig. 1(B)].

To estimate the transfer rates of single congeners from dam tissues to milk, we have calculated the ratio between the milk congener concentrations and those measured in dam tissues and we considered this parameter as the Transfer ratio (Tr). Higher $\operatorname{Tr}$ values indicate higher transfer rates. The $\operatorname{Tr}$ from adipose tissue to milk evidenced a slightly higher transfer rate for PCB 153 in comparison with PCB 138 and 180 [Fig. 2(A)]. Moreover starting from our previously published data (Cocchi et al., 2009), the Tr calculated from maternal liver and brain to milk has demonstrated that these tissues release more easily the congeners in comparison to adipose tissue [Fig. 2(B)]. Unlike adipose tissue, where PCB 153 was released more readily, liver and brain set PCB 138 free more efficiently to maternal milk (Fig. 2). Moreover, comparing the Trs of liver and brain it is evident that PCB153 is retained more effectively by brain than liver [Fig. 2(B)].

The transfer of dosed compounds to offspring during lactation was analyzed calculating $\mathrm{Tr}$ from milk to brain and liver of pups. The results demonstrated that PCB 153 is more efficiently transferred through maternal milk to pups in comparison to PCB 138 and 180, and that liver showed a relatively higher retention of congeners than brain (Fig. 3).

\section{Hepatic Total Cytochrome P450 Content and CYP Isoform Expression in Maternal and Offspring Tissues}

Total CYP contents were investigated in maternal and offspring liver at time points PN12, PN21, and PN60 and the results are shown in Figure 4. Constitutive CYP contents remained unchanged in dams, suckling, weaning, and adult offspring. RM administration to dams from GD15 to PN21 produced an increase in total mothers' hepatic P450 concentration in comparison with their respective controls, even if it resulted to be statistically significant $(P<0.05)$ only at PN21 and PN60. Statistical analysis evidenced that at PN60, the increment was significant $(P<0.005)$ with respect to the contents measured in treated PN12 and PN21 dams [Fig. 4(A)]. 

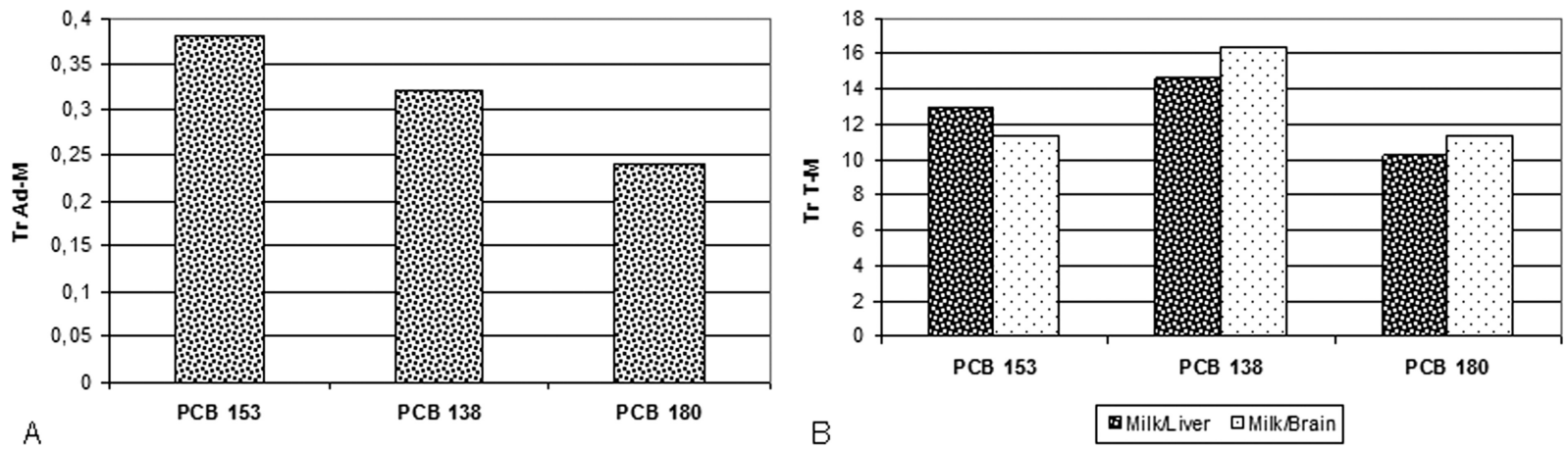

Fig. 2. Transfer ratio ( $\mathrm{Tr}$ ) of each $\mathrm{PCB}$ congener from maternal tissues to milk calculated as ratio between congener concentrations in milk and dam tissues. (A) Transfer ratio from Adipose tissue into the milk ( $\operatorname{Tr} \mathrm{Ad}-\mathrm{M})$. (B) Transfer ratio from dam tissues (liver and brain) into the milk ( $\operatorname{Tr} \mathrm{T}-\mathrm{M})$. ( $n=5$ dams).

An increase of hepatic P450 total contents was observed as well in offspring in response to maternal treatment at the three time points [Fig. 4(B)]. The statistical analysis demonstrated that the increment versus the corresponding controls was significant $(P<0.001)$ only during lactation and weaning, and that among PCB exposed pups a significant peak $(P<0.001)$ of hepatic $\mathrm{P} 450$ total level was registered in weaning offspring [Fig. 4(B)].

To assess changes in protein expression levels of both hepatic and cerebral CYP1A1/2 and CYP2B1/2 isoforms in mothers and offspring, we performed immunoblot analyses.

The polyclonal antibody against CYP1A1/2 recognized a single band in control liver samples of all experimental groups, most likely ascribable to CYP1A2, known as hepatic constitutive isoform (Hu and Bunce, 1999; Spatzenegger et al., 2000). In details, a faint but detectable basal expression of CYP1A2 was evidenced in microsomes collected from control liver dams. A strong induction was observed in response to RM treatment at all considered time points, mainly referable to the inducible CYP1A1 hepatic isoform (Hu and Bunce, 1999; Spatzenegger et al., 2000) [Fig. 5(A)]. As revealed from densitometric analysis, CYP1A induction increased from lactation (PN12) to weaning (PN21) time points of $\sim 20 \%$, reaching at PN60 a level of $280 \%$ over the expression of lactating treated dams [Fig. 5(A)].

The polyclonal antibody against CYP2B1/2 identified in dam liver a single constitutive and PCB-induced CYP2B expression [Fig. 5(B)]. The densitometric analysis demonstrated that the induction in treated dams was statistically significant $(P<0.005$ for PN12 and $P<0.05$ for PN21 and PN60) and time-dependent. In liver of PCB-treated dams, CYP2B protein levels were about 200\% higher than the corresponding control values during lactation and weaning period, while they increased remarkably reaching values $750 \%$ over control at PN60.

When examined in control and transplacentally/lactationally exposed offspring as a function of age, both
CYP1A and CYP2B protein levels exhibited a similar pattern of expression. Basal levels of both CYP families remained nearly unchanged in liver of suckling (PN12), weaning (PN21), and adult (PN60) control offspring while in exposed progeny the protein expression levels exhibited a significant increment at PN12 (about 70\% over control) and especially at PN21 (about 270\% over control; see legend of Fig. 6 for $P$ values). Contrary to dams, in adult PCB offspring CYP protein levels showed a decline up to values not significantly different from those of the control (Fig. 6).

On the whole, the time courses of CYP1 and 2 family members induction in dams and offspring resemble the profile of hepatic P450 total contents.

Differently from livers, in whole brains the low constitutive levels of both CYP1A and CYP2B isoforms have not been induced by treatment with the PCB mixture both in dams and offspring (data not shown).

Interestingly, while in liver of dams the levels of induction undergo a drastic increase at PN60, a time point far

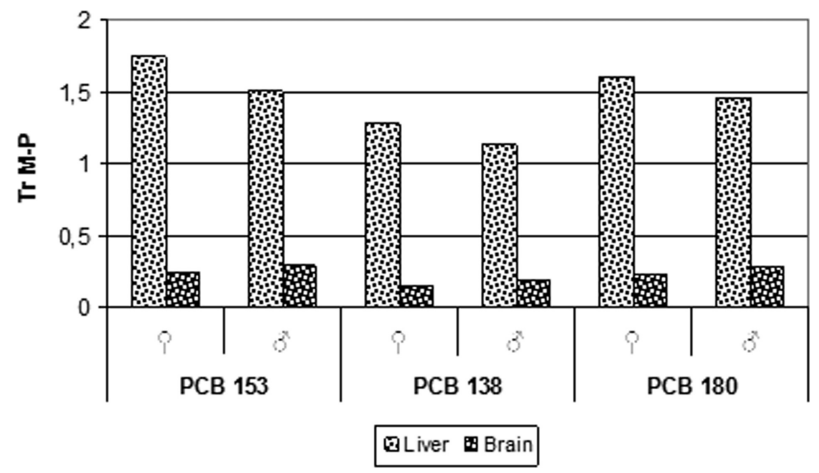

Fig. 3. Transfer ratio of each PCB congener from milk ( $n=$ 5 dams) to liver and brain of single male ( $n=3$ for each dam) and single female ( $n=3$ for each dam) pups at PN12 (Tr M-P) calculated as ratio between congener concentrations in milk and in pup tissues. 

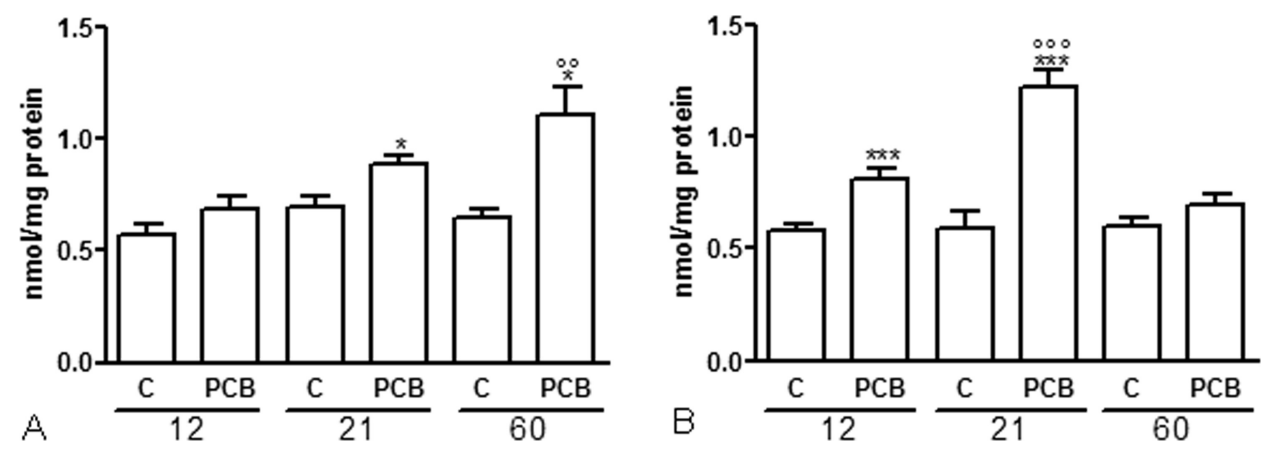

Fig. 4. Hepatic cytochrome P450 total content at time points PN12, PN21, and PN60 in single control $(C)$ and treated dams $(n=5$; A) and in their respective offspring singly evaluated ( $n=3$ males and $n=3$ females; B). Statistical differences were evaluated with Duncan's multiple comparison after ANOVA. All values are represented as mean \pm SEM. Significant difference from control * $(P<0.05)$; ${ }^{\star \star \star}(P<0.001)$. Significant difference among treated groups ${ }^{\circ \circ}(P<0.005) ;{ }^{\circ 00}(P<0.001)$.

away from the last $\mathrm{PCB}$ administration that occurred at weaning, in liver of offspring the CYP isoform induction is consistent but transient and restricted to lactation and weaning periods (Fig. 7). In particular, CYP expression time course in dams correlates with PCB levels measured in liver at PN12, PN21, and PN60, whereas CYP expression time course in offspring shows a latency period as CYP1A and CYP2B induction becomes maximal at weaning (PN21) when PCB concentrations in liver start to decrease (see Cocchi et al., 2009).

\section{DISCUSSION}

The present study, representing an extended investigation of a previous multitasked one, demonstrates that treatment with a biologically relevant reconstituted PCB mixture of dams during pregnancy and lactation results in an effective transfer of PCBs to neonates and in a significant responsiveness of hepatic CYP in both mothers and offspring.

PCB congeners selected for our RM are present at high concentration in the maternal mesenteric adipose reserves,
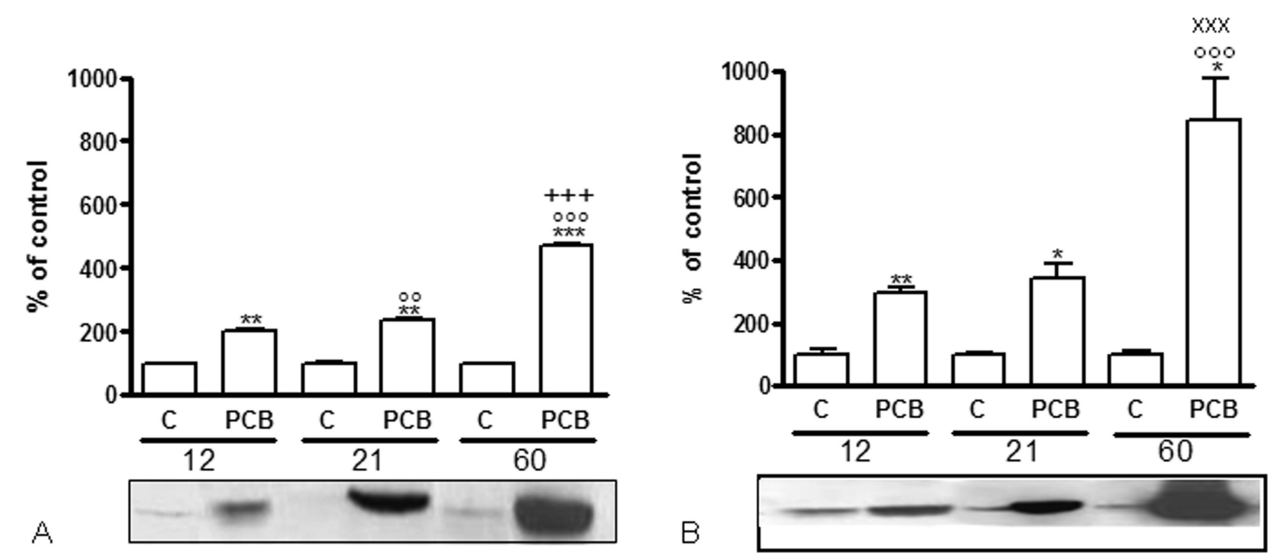

Fig. 5. Representative Western blot and densitometric analysis of CYP1A (A) and CYP2B (B) isoforms at each experimental point in control $(C)$ and treated maternal liver. Quantification was performed by computerized densitometric analysis of immunoreactive bands on at least three separate blots for each sample ( $n=5$ dams for each group) to account for blot to blot variation. Statistical differences were evaluated by Kruskal-Wallis test coupled with Dunn's test. All values are represented as mean \pm SEM. Significant difference from control * $(P<0.05) ;{ }^{* *}(P<0.005) ;{ }^{* * *}(P<0.001)$. Significant difference among treated groups ${ }^{\circ}(P<0.005) ;{ }^{\circ}(P<0.001)$. Significant difference between PN60 and PN21 treated groups $+++(P<0.001)$. Significant difference between PN60, PN21, and PN12 treated groups $\mathrm{XXX}(P<0.001)$. 

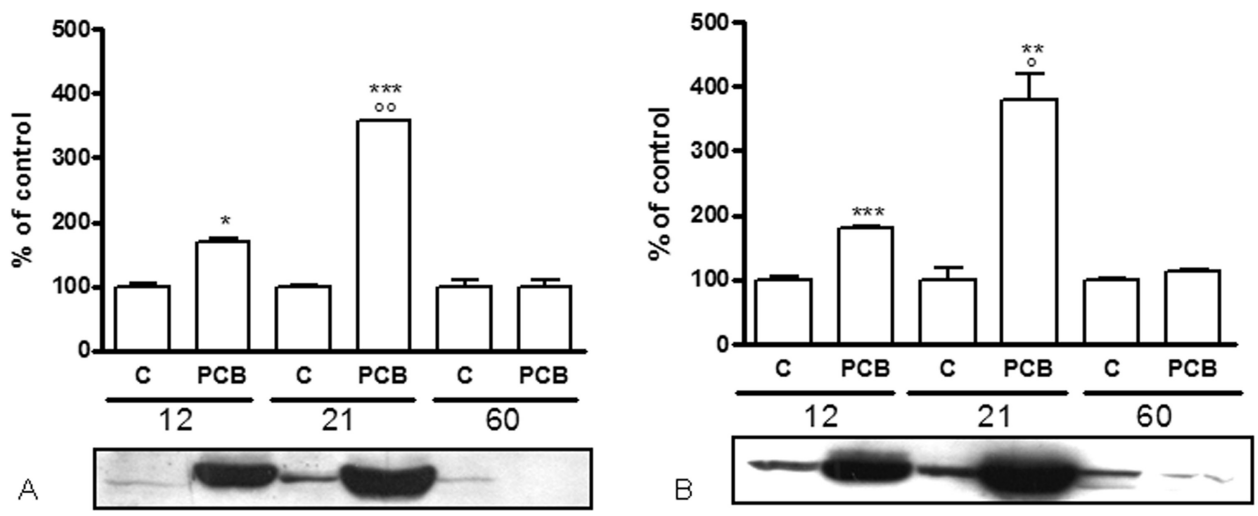

Fig. 6. Representative Western blot and densitometric analysis of CYP1A (A) and CYP2B (B) isoforms at each experimental point in control (C) and exposed offspring liver. Quantification was performed by computerized densitometric analysis of immunoreactive bands on at least three separate blots for each sample $(n=3$ males and $n=3$ females for each dam) to account for blot to blot variation. Statistical differences were evaluated by Kruskal-Wallis test coupled with Dunn's test. All values are represented as mean \pm SEM. Significant difference from control * $(P<0.05)$; ${ }^{* *}(P<0.005) ;{ }^{\star * \star}(P<0.001)$. Significant difference among treated groups ${ }^{\circ}(P<0.05) ;{ }^{\circ}(P<0.005)$.

as expected from their high degree of chlorination. Despite this, only 4-fold lower concentration was found in maternal milk. Several studies demonstrate that under environmental exposure circumstances, the levels of organohalogens in human milk reflect, to a great extent, adipose tissue levels, a reservoir that is built up during several years and is mobilized during lactation (Jensen, 1991). This behavior proves the tendency of highly chlorinated PCB congeners to remain unmetabolized and pass through the rich lipid biological matrices. Indeed, many studies have evidenced that the profile of PCB congeners measured in milk samples of marine mammals and humans is dominated by hexachlorobiphenyl (i.e., PCB153, 138) and heptachlorobiphenyl congeners (i.e., PCB170, 180; Miranda et al., 2009; Someya et al., 2010), which beside their lipophilicity are moved from adipose tissue and vertically transferred from mother to newborn through breast milk. From our transfer rate data, it appears that liver and brain free more easily these lipophilic congeners in comparison to adipose tissue. Nevertheless adipose tissue, containing a PCB concentration an order of magnitude higher than that found in liver and brain (Cocchi et al., 2009), gives a higher contribution to the amount of PCBs detected in maternal milk.

Even though it is generally believed that milk with its high lipid content is the most significant via of offspring contamination, it has to be noticed that PCB transfer through placenta can play an important role (Dekoning and Karmaus, 2000; Bonfanti et al., 2009). In our previous article, we evidenced relatively high Trs (ranging from 0.5 to 0.95 ) from placenta to brain and liver of fetuses at GD20. It is noteworthy that at GD20 we have obtained a Tr from placenta to fetal brain higher than that from placenta to fetal liver (mean values of 0.8 vs. 0.6 , respectively), while in this study the $\mathrm{Tr}$ from milk to pup liver is higher than that from milk to pup brain (mean values of 1.5 vs. 0.22 , respectively). These results suggest that during fetal period, brain is a target organ for PCB accumulation, while during neonatal period liver gains the role of depot organ. These data are consistent with those described by Curran et al. (2011) in Ahr ${ }^{b 1}$ Cypla2 $(+/+1)$ mice strain. The high $\mathrm{Tr}$ from milk to pup liver could be the consequence of an efficient gastrointestinal absorption of individual congeners in neonate rats. To this end, a study reported that the individual congeners and their mixtures are readily absorbed from the gastrointestinal tract of rodents and monkeys to levels varying between 66 and $96 \%$ in relation to degree of chlorination (WHO, 2000). Considering the high efficiency of the PCB gastrointestinal absorption, current neonatal exposures continue to raise serious concerns regarding potential health effects on developing infants, even if PCB levels in human milk have decreased consequently to their ban (Abballe et al., 2008).

In spite of the demonstrated transplacental transfer and disposition of PCB congeners in liver and brain of fetuses, fetal CYP1A and CYP2B isoforms were not sensitive to the measured PCB levels (Bonfanti et al., 2009). Instead, in this study we provided evidence that the effective transfer of PCB congeners from milk to pup liver goes along with sensitivity of the hepatic CYP isoforms toward inductive effects of chemicals tested. Though the levels of PCB126 congener were not analytically measured in milk, the increase in the expression of hepatic CYP1A1 isoform in suckling pups indicates that lactational transfer of this congener occurs.

When CYP temporal expression of dams and their offspring are compared, there are differences in CYP response as the induction of CYP1A1/2 and CYP2B1/2 both in mother and offspring is dependent on lactational transfer of 


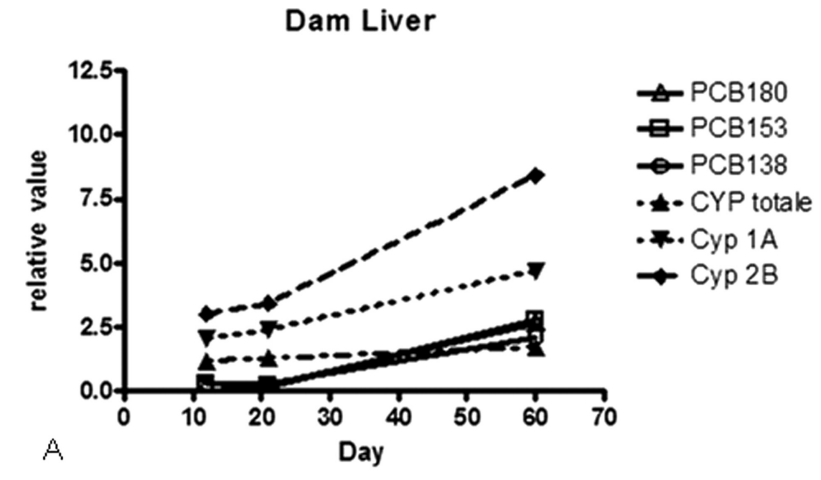

Pup Liver

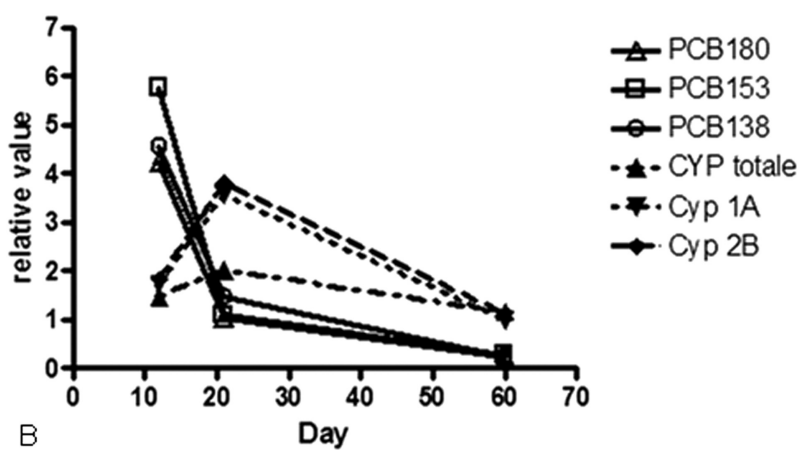

Fig. 7. Comparison between hepatic cytochrome P450 (total content, CYP1A and CYP2B isoform expression levels) and $\mathrm{PCB}$ congener concentration in treated dams $(\mathrm{A})$ and pups (B). Since the reference scale for each considered parameter was different, experimental data were normalized to obtain the relative value reported on $y$-axis. Relative value referred to PCB congeners represents the ratio between single congener concentration and total PCB concentration. Relative value referred to CYP total content and CYP isoform expression represents the ratio between each value obtained in PCB-treated samples and their respective control value at each time point.

PCBs. Although CYP response in dams acts as a faithful biomarker of exposure, varying according to the elimination of PCBs through milk, CYP isoform levels in suckling and weaning offspring do not match with the hepatic PCB concentrations showing a delay in CYP response probably related to the immaturity of this metabolic system in rat neonates (Blake et al., 2005). Nevertheless, in adult offspring CYP isoform expression levels return to be not significantly different from those of the control, reflecting accurately the low PCB concentration in liver. In fact, in rodents offspring the PCB concentrations in liver and brain decline after weaning (Curran et al., 2011) mainly owing to dilution in the increasing fat deposits, as demonstrated also in children (WHO, 2000).

A noteworthy result of this study is that the developmental responsiveness of the tested CYP isoforms to PCB exposure in offspring liver is transient and does not alter persis- tently the expression of these enzymes. A different result was found in rat offspring prenatally exposed to other chemicals such as deltametrhin and lindane as these lipophilic compounds are able to imprint an altered expression of cerebral and hepatic CYPs up to the adulthood (Jorhi et al., 2006, 2008). The persistence in the increase in CYP mRNA expression is explained by the authors through the lactational transfer of these lipophilic compounds to offspring during the critical period of neurodevelopment. This persistent alteration in postnatal development of CYPs has been proposed to be involved in developmental neurotoxicity of these pesticides, since it has been shown that CYPs (i.e., CYP 2E1) are involved in physiological functions in brain such as dopaminergic neurotransmission in the substantia nigra, possibly by participating in dopamine metabolism (Nissbrandt et al., 2001).

An unexpected result in our study regards the brain CYP sensitivity, because both in mothers and offspring the constitutive CYP1A and CYP2B isoforms were not responsive to the induction by PCB mixture even if in this organ detectable levels of each congener were accumulated (Cocchi et al., 2009).

Nevertheless, as reported in our previous article (Colciago et al., 2009) the RM PCB congeners accumulated in brain during lactation and weaning were able to cause persisting alteration in the control of the reproductive system: in particular, the dimorphic hypothalamic expression of both aromatase and the $5 \alpha$-Rs, two enzymes of testosterone metabolism, was altered by the treatment. The puberty onset in female-treated pups occurred earlier than in control animals without cycle irregularity, while testicular descent in males was delayed. A slight but significant impairment of sexual behavior and an important alteration in memory retention were also noted specifically in males. Furthermore, there are numerous reports indicating that $\mathrm{PCBs}$ induce subtle and long-lasting neurological damages such as impairment of motor and learning abilities (Ulbrich and Stahlman, 2004; Piedrafita et al., 2008).

Since developmental neurotoxicity of our PCB RM was not found to be correlated with an altered expression of CYPs in brain of offspring prenatally and lactationally exposed, an involvement of the local metabolizing enzymes in PCB induced toxicity previously evidenced should be excluded. Nevertheless, literature data reported either a selective concentration of noncoplanar PCBs (i.e., PCB153) in several areas of weanling rat brain, such as corpus callosum, internal and external capsules, medial lemniscus, tegumentum of mesencephalon and metencephalon and cerebellar peduncles (Saghir et al., 2000), as well as a localized expression of specific CYP isoforms (i.e., CYP $1 \mathrm{~A} 1 / 2$ ) in rodent neurons of cerebral cortex, ippocampus, Purkinje and granule cell layers of cerebellum (Chinta et al., 2005). Unfortunately, we used whole brain for sampling, thus losing the opportunity to highlight localized effects within specific brain structures or cell types. 
In conclusion, the present study contributes to extend the knowledge about vertical transfer of highly chlorinated PCBs by maternal milk and responsiveness of hepatic and cerebral CYP isoforms to these potent inducers during rat development. In lactationally exposed offspring, the alteration of liver CYP isoform levels indicates a transcriptional regulation mediated, as known, by a binding of these environmental chemicals to cytosolic receptors (Bandiera, 2001), evidencing their biological activity. On the other hand, in brain the lack of CYP induction does not exclude the susceptibility of this organ to PCBs during postnatal development as reported in our previous articles (Cocchi et al., 2009; Colciago et al., 2009). These considerations suggest the presence of a different pathway in the neurotoxicity of these compounds. Indeed, in literature PCB neurotoxicity has been related to many different mechanisms: it has been proposed their ability to alter patterns of ryanodine receptors (RyRs) expression in brain, altering calcium flux in excitable cells (Pessah et al., 2010; Kim et al., 2011); it has been suggested pollutants ability to induce an inflammatory phenomena in neurons, altering their function and inducing neurotoxicity (Banks and Lein, 2012); lastly, PCB are able to disrupt some epigenetic markers related to chromatin remodeling favoring or silencing the translation of genes important for neuronal development and function (Casati et al., 2012).

The relevance of the results previously obtained in our multitasked studies to findings reported in this manuscript is related to the confirmed transfer of PCBs from mother to offspring during gestation and lactation. These data corroborate that the perinatal period of life is at high risk for environmental damages on public health.

The authors declare that there is no conflict of interest that would prejudice the impartiality of this scientific work.

\section{REFERENCES}

Abballe A, Ballard TJ, Dellatte E, di Domenico A, Ferri F, Fulgenzi AR, Grisanti G, Iacovella N, Ingelido AM, Rainer M, Miniero R, Porpora MG, Risica S, Ziemacki G, De Felip E. 2008. Persistent environmental contaminants in human milk: Concentrations and time trends in Italy. Chemosphere 73:S220-S227.

Bachour G, Failing K, Georgii S, Elmadfa I, Brunn H. 1998. Species and organ dependence of PCB contamination in fish, foxes, roe deer, and humans. Arch Environ Contam Toxicol 35:666-673.

Bandiera SM. 2001. Cytochrome P-450 enzymes as biomarkers of PCB exposure and modulators of toxicity. In: Robertson LW, Hansen LG, editors. PCBs: Recent Advances in Environmental Toxicology and Health Effects. Lexington, Kentucky: University Press of Kentucky, pp 185-192.

Banks CN, Lein PJ. 2012. A review of experimental evidence linking neurotoxic organophosphorus compounds and inflammation. Neurotoxicology 33:575-584.

Blake MJ, Castro L, Leeder JS, Kearns GL. 2005. Ontogeny of drug metabolizing enzymes in the neonate. Semin Fetal Neonatal Med 10:123-138.
Bonfanti P, Colombo A, Villa S, Comelli F, Costa B. 2009. The effects of accumulation of an environmentally relevant polychlorinated biphenyl mixture on cytochrome P450 and P-glycoprotein expressions in fetuses and pregnant rats. Chemosphere 75:572-579.

Casati L, Sendra R, Colciago A, Negri-Cesi P, Berdasco M, Esteller M, Celotti F. 2012. Polychlorinated biphenyls affect histone modification pattern in early development of rats: A role for androgen receptor-dependent modulation? Epigenomics 4:101-112.

Chinta SJ, Kommadi RP, Turman CM, Strobel HW, Ravindranat V. 2005. Constitutive expression and localization of cytochrome P-450 1A1 in rat human brain: Presence of a slice variant form in human brain. J Neurochem 93:724-736.

Cocchi D, Tulipano G, Colciago A, Sibilia V, Pagani F, Vigano D, Rubino T, Parolaio D, Bonfanti P, Colombo A, Celotti F. 2009. Chronic treatment with polychlorinated biphenyls (PCB) during pregnancy and lactation in the rat, Part 1: Effects on somatic growth, growth hormone-axis activity and bone mass in the offspring. Toxicol Appl Pharmacol 237:127-136.

Colciago A, Casati L, Mornati O, Vergoni AV, Santagostino A, Celotti F, Negri-Cesi P. 2009. Chronic treatment with polychlorinated biphenyls (PCB) during pregnancy and lactation in the rat, Part 2: Effects on reproductive parameters, on sex behavior, on memory retention and on hypothalamic expression of aromatase and $5 \alpha$-reductases in the offspring. Toxicol Appl Pharmacol 239:46-54.

Curran CP, Vorhees CV, Williams MT, Genter MB, Miller ML, Nebert DW. 2011. In utero and lactational exposure to a complex mixture of polychlorinated biphenyls: Toxicity in pups dependent on the Cyp1a2 and Ahr genotypes. Toxicol Sci 119:189-208.

Dekoning EP, Karmaus W. 2000. PCb exposure in utero and via breast milk. A review. J Expo Anal Environ Epidemiol 10:285-293.

Eriksson P, Fischer C, Fredriksson A. 2006. Polybrominated diphenyl ethers, a group of brominated flame retardants, can interact with polychlorinated biphenyls in enhancing developmental neurobehavioral defects. Toxicol Sci 94:302-309.

Filho KCM, Metcalfe CD, Metcalfe TL, Muelbert MMC, Robaldo RB, Martinez PE, Colares EP, Bianchini A. 2009. Lactational transfer of PCBs and chlorinated pesticides in pups of southern elephant seals (Mirounga leonine) from Antarctica. Chemosphere 75:610-616.

Gallenberg LA, Vodicnik MJ. 1989. Transfer of persistent chemicals in milk. Drug Metab Rev 21:277-317.

Hu K, Bunce NJ. 1999. Metabolism of polychlorinated dibenzop-dioxins by rat liver microsomes. J Mol Biochem Toxicol 13:307-315.

Jensen AA. 1991. Transfer of chemical contaminants into human milk. In: Jensen AA, Slorach SA, editors. Chemical Contaminants in Human Milk. Boca Raton (FL), CRC Press. pp 9-19.

Jorhi A, Dhawan A, Singh RL, Parmar D. 2006. Effect of prenatal exposure of deltamethrin on the ontogeny of xenobiotic metabolizing cytochrome P450s in the brain and liver of offsprings. Toxicol Appl Pharmacol 214:279-289.

Jorhi A, Yadav S, Dhawan A, Parmar D. 2008. Responsiveness of cerebral and hepatic cytochrome $\mathrm{P} 450$ in rat offspring prenatally exposed to lindane. Toxicol Appl Pharmacol 231:10-16. 
Kim KH, Bose DD, Ghogha A, Riehl J, Zhang R, Barnhart CD, Lein PJ, Pessah IN. 2011. Para- and ortho-substitutions are key determinants of polybrominated diphenyl ether activity toward ryanodine receptors and neurotoxicity. Environ Health Perspect 119:519-526.

Lanting CI, Huisman M, Muskiet FA, van der Paauw CG, Essed CE, Boersma ER. 1998. Polychlorinated biphenyls in adipose tissue, liver, and brain from nine stillborns of varying gestational ages. Pediatr Res 44:222-225.

Lowry OH, Rosebrough NJ, Farr AL, Randall RJ. 1951. Protein measurement with the Folin phenol reagent. J Biol Chem 193:265-275.

Miranda Filho KC, Metcalfe CD, Metcalfe TL, Muelbert MMC, Robaldo RB, Martinez PE, Colares EP, Bianchini A. 2009. Lactational transfer of PCBs and chlorinated pesticides in pups of southern elephant seals (Mirounga leonine) from Antarctica. Chemosphere 75:610-616.

Mitsuro A, Hiroshi S, Ichiro W. 2005. Transfer of polychlorinated biphenyls (PCBs) to newborn infants through the placenta and mothers' milk. Arch Environ Contam Toxicol 14:51-57.

Nissbrandt H, Bergquist F, Jonason J, Engherg G. 2001. Inhibition of cytochrome P450 2E1 induces an increase in extracellular dopamine in rat substantia nigra: A new metabolic pathway? Synapse 40:294-301.

Omura T, Sato R. 1964. The carbon monoxide-binding pigment of liver microsomes. I. Evidence for its hemoprotein nature. J Biol Chem 239:2370-2378.

Pessah IN, Cherednichenko G, Lein PJ. 2010. Minding the calcium store: Ryanodine receptor activation as a convergent mechanism of PCB toxicity. Pharmacol Ther 125:260-285.

Piedrafita B, Erceg S, Cauli O, Monfort P, Felipo V. 2008. Developmental exposure to polychlorinated biphenyls PCB153 or PCB126 impairs learning ability in young but not in adult rats. Eur J Neurosci 27:177-182.

Ramos L, Hernandez LM, Gonzalez MJ. 1997. Variation of PCB congener levels during lactation period and relationship to their molecular structure. Arch Environ Contam Toxicol 33:97-103.

Rifkind AB. 2006. CYP1A in TCDD toxicity and in physiologyWith particular reference to CYP dependent arachidonic acid metabolism and other endogenous substrates. Drug Metab Rev 38:291-335.

Roegge CS, Schantz SL. 2006. Motor function following developmental exposure to PCBS and/or MEHG. Neurotoxicol Teratol 28:260-277.

Safe S. 1994. Polychlorinated biphenyls (PCBs): Environmental impact, biochemical and toxic responses, and implication for risk assessment. Crit Rev Toxicol 24:87-149.

Saghir SA, Hansen LG, Holmes KR, Kodavanti PRS. 2000. Differential and non-uniform tissue and brain distribution of two distinct ${ }^{14} \mathrm{C}$-hexachlorobiphenyls in weanling rats. Toxicol Sci 4:60-70.

Schuetz EG, Wrighton SA, Safe SH, Guzelian PS. 1986. Regulation of cytochrome P-45Op by phenobarbital and phenobarbital-like inducers in adult rat hepatocytes in primary monolayer culture and in vivo. Biochemistry 25:1124-1133.

Someya M, Ohtake M, Kunisue T, Subramanian A, Takahashi S, Chakraborty P, Ramachandran R, Tanabe S. 2010. Persistent organic pollutants in breast milk of mothers residing around an open dumping site in Kolkata, India: Specific dioxinlike PCB levels and fish as a potential source. Environ Int 36:27-35.

Spatzenegger M, Horsmans Y, Verbeeck RK. 2000. Differential activities of CYP1A isozymes in hepatic and intestinal microsomes of control and 3-methylcholanthrene induced rats. Pharmacol Toxicol 86:71-77.

Ulbrich B, Stahlman R. 2004. Developmental toxicity of polychlorinated biphenyls (PCBs): A systematic review of experimental data. Arch Toxicol 78:253-268.

WHO. 2000. Polychlorinated biphenyls. In: Air Quality Guidelines. World Health Organization, Regional Office for Europe, Copenhagen, Denmark, 2nd ed. Chapter 5.10. pp 1-22. 